\title{
Towards a methodical approach to implement biomimetic paradigms in the design of robotic systems for space applications
}

\author{
C. Menon, ${ }^{a *}$ N. Lan ${ }^{\mathrm{b}}$ and D. Sameoto ${ }^{\mathrm{a}}$ \\ ${ }^{a}$ Menrva Group, School of Engineering Science, Simon Fraser University, Burnaby V5A 1S6, Canada; ${ }^{b}$ Ursa Minor Space \\ and Navigation B.V., 2629 HD Delft, The Netherlands
}

(Received 20 August 2008; final version received 3 January 2009)

\begin{abstract}
Biomimetic design is considered a promising source of novel solutions to problems in space engineering and robotics in particular. With the maturing of this discipline, however, a need is identified: a more systematic approach to its application to reduce the element of chance in the design of biomimetic systems. A methodology is proposed to address this concern and provide a basis for further development of biomimetic design procedures. The application of this process is illustrated through case studies of ongoing biomimetics research with relevance to space robotics in the form of climbing robots utilising synthetic dry adhesives.
\end{abstract}

Keywords: biomimetics; robotics; space engineering; methodology

\section{Introduction}

Biomimetics, the process of extracting concepts from biological organisms and applying them in engineering, is a maturing discipline. Currently, most biomimetically designed systems rely on expert input to determine what aspects of and in what way biological systems should be translated into an engineering context. Given that biomimetics may be applied in many fields of engineering and ideas may be taken from the whole range of natural organisms, it is not surprising that this emerging discipline has transformed concepts to engineered systems in a relatively ad hoc manner so far. In the maturing of this field, methodical approaches are desirable. While work in these areas is ongoing, a main focus has been on the early-stage process of identifying biological solutions that may be studied to address engineering problems in a methodical manner. The authors have felt that approaches that may be applied to the entire biomimetic design process, including detailed system design and implementation, should also be addressed. Furthermore, while current and nearterm biomimetic design is likely to continue to be expertdriven, whether methodical or ad hoc, it is felt that potential for automation should be considered in the development of generalised approaches. These are, of course, highly challenging goals and as such this work presents preliminary qualitative suggestions for further progress towards a generalised methodology. Through development of general biomimetic design methodology, it is envisaged that the biomimetic design of space systems will be facilitated.
A case study involving current hardware developments is presented to illustrate the suggested framework. This case study specifically addresses the design of space systems using the proposed methodology for general biomimetic system design. With advances in this field, it is hoped that generalised biomimetic research carried out by multidisciplinary groups may be facilitated and researchers may be able to turn to biomimetics as a standard option when faced with the task of finding a novel solution to a problem.

\section{Terminology}

Goals of the proposed approach to a generalised, comprehensive methodology for biomimetic system design include the enabling of both systematic search for solutions at all stages as well as potential for automation. A principle identified challenge relating to these goals is the decoupling of aspects of biological systems to be considered for translation so that they may be considered and combined individually. While such decoupling may be impossible to achieve completely, the following terminology is suggested such that it may be implemented to some extent. An example of use is shown in Figure 1.

Function: A capability of the system to perform a particular task, e.g. to control altitude in a gravitationally bound fluid.

Characteristic: A distinctive quality of a system in relation to its function, e.g. lightness, robustness, autonomy and efficiency.

*Corresponding author. Email: cmenon@sfu.ca 


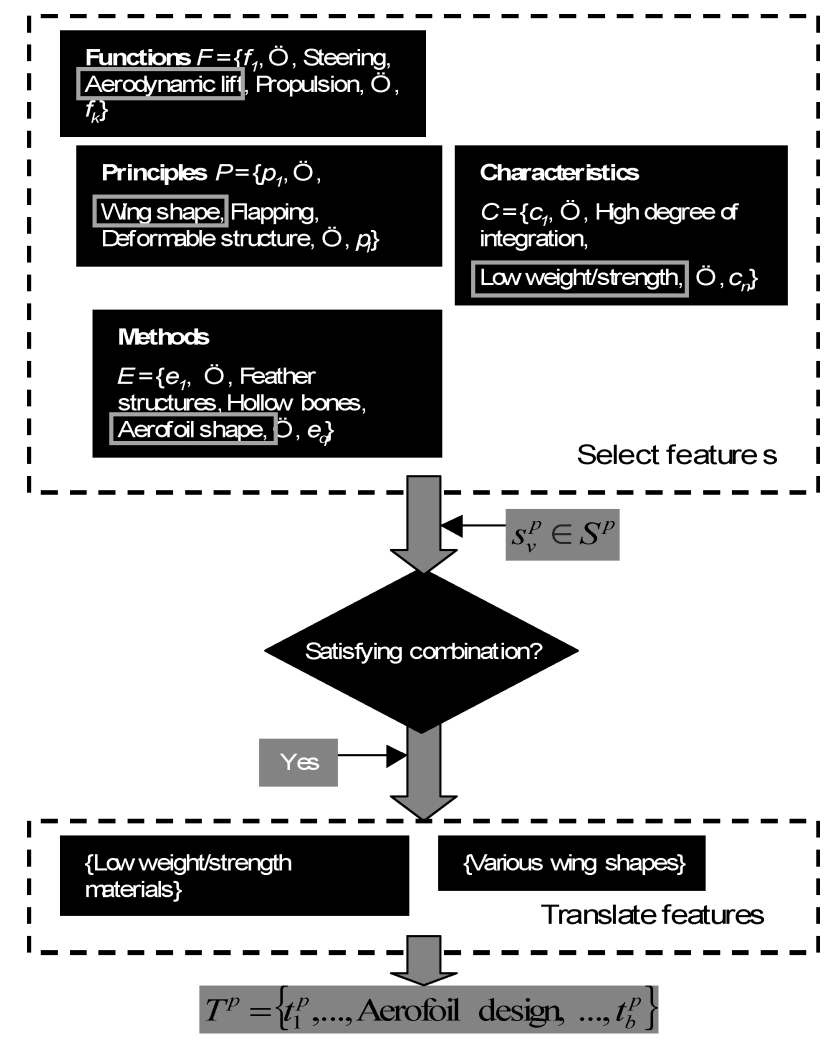

Figure 1. Single iteration of the system abstraction processes as shown in Figure 1(c). The boxes in green denote the particular element $s_{v}^{p} \in S^{p}$.

Principle: A means by which a system is able to perform a function with a certain characteristic or characteristics, e.g. a physical principle such as creating aerodynamic lift with an aerofoil shape or creating adhesion through contact splitting.

Method: A specific way in which a principle or characteristic is implemented, e.g. through use of particular materials or structures

Features: The functions, principles, characteristics and methods of a biological system.

Level of abstraction: Qualitatively, the level of abstraction of a biomimetically engineered system is a measure of how dissimilar it is to its biological analogue. A method of measurement of level of abstraction may be to compare the principles and characteristics employed in each system plus the methods used to implement these principles and characteristics. We define that a high correlation from this comparison indicates a low level of abstraction and vice versa. This metric might be considered in relation to a biomimetic system as a whole or the translation of a single function, for example.

\section{Biomimetics for space}

\subsection{Challenges of space environment}

In comparison to most terrestrial environments, space poses specific challenges to robotic systems. Particular differing physical factors range from the radiation and atomic oxygen environment in earth orbits to harsh extraterrestrial environments with extreme atmospheric conditions. Not only do these factors require mitigation through design, but also most space robotic systems have little chance of being repaired once in situ, thus making their reliability and robustness of paramount importance. This isolation of spaceborne robotic systems, with respect to the impact on the mission of communication with terrestrial operators, further motivates the implementation of autonomous capabilities. Requirements for these capabilities may be demanding, given that such systems must operate robustly in unpredictable or unstructured environments. Furthermore, with future missions, perhaps involving networks of discrete robotic agents, greater abilities in terms of autonomous communication and cooperation will be enabling. Perhaps the greatest impact on space systems derives from the high cost of access to space; lower costs can often be achieved through considerable reduction in mass, volume and power consumption.

\subsection{The case for biomimetics in space}

A common statement supporting the usefulness of biomimetics in engineering is that biological organisms face many of the same challenges as engineering systems do. Space presents an environment radically different from that faced by all known organisms, such that biomimetics might be thought to be inappropriate in these terms. However, it can be seen that while direct analogies between space systems and biological organisms might be less common, many species exhibit qualities that are highly desirable in space system design, such as robustness, light weight, low volume and power, autonomy, adaptability and self-repair. In some cases, it may even be seen that biomimetic solutions can even take advantage of the peculiarities of extraterrestrial environments in novel ways (e.g. Martian atmospheric conditions allow consideration of passively propelled rovers inspired by tumbleweed (ESA 2005) and insect-inspired flapping flight (Michelson and Naqvi 2003)).

Previous studies performed by the authors in collaboration with academic and industrial partners, have highlighted several fields of space technology development where biomimetics might be profitably applied (ESA 2005; Menon et al. 2007a, 2007b). This wide interest in biomimetics in space motivates the continuing growth of biomimetics as an increasingly systematic and distinct discipline for the design of novel high-performance space systems. Several examples are available of biomimetic systems for use in space studied by the authors in collaboration with various research groups and spanning a range of applications applicable to robotics, each with particular advantageous characteristics. These include hydraulic actuators based on the actuation system of the spider (Menon and Lira 2006), an integrated actuated joint based on the human knee (ESA 
2006) and structurally embedded strain sensors based on those found in insect exoskeletons (Vincent et al. 2007). Further examples of potential areas in space engineering where biomimetics may be applied include life and physical sciences instrumentation, telepresence, tribology, pyrotechnics, environmental control and life support (ECLS) and in situ resource utilisation (ISRU) (Biomimetic Engineering for Space Applications 2006).

\section{Towards a general methodology}

\subsection{Problem definition}

In the following sections, we present suggestions for the design of such a methodology for performance of biomimetic system design specifying what is desired, identifying the challenges inherent in such an undertaking and reviewing the state of previous work in this area. While it is deemed to be most useful to make these suggestions in the context of biomimetic design as a whole, it is envisaged that such development would encompass use in space system design and promote use in this field.

The origin of a transfer of ideas may come from biology with the identification of an attractive characteristic or attribute in an organism inspiring a search for an engineering application. On the other hand, a weakness in engineering solutions may be identified, at which point one might turn to nature to suggest a novel approach. That initiation may come from either engineering or biology and wide-ranging knowledge (whether from experts or databases) from both fields is a crucial first requirement for systematic biomimetics. Either way, expert knowledge provided from both engineering and biological study is critical at this stage, in identifying the particular means by which organisms perform their functions and matching these potential solutions appropriately to problems in engineering.

Translation of designs from a biological to an engineering context is perhaps the most important and most difficult part of the biomimetic process. As suggested previously, biomimetics does not seek to merely copy biological systems, but to apply aspects of their design to achieve functions in an engineered system with different objectives and constraints. That biomimetics may be applied to several different space technology areas also increases the complexity of generalising a design process. In addition, the principles and methods employed in various biological systems and subsystems are highly variable. Furthermore, new technologies are offering an increasing number of previously unavailable options in creating engineered analogues of biological systems as for example in the potential for use of shape memory alloys (SMAs) or electro-active polymers (EAPs) in mimicking the action of muscles in miniaturised systems. The solution space of options for translation from a biological to an engineering system can therefore be seen to be large. Given the large sets of potential problem-to- solution matches and potential translations of solution to problem, a biomimetic methodology would ideally allow some automation of these processes.

An additional barrier to consensus throughout the biomimetic community is that not only are many different disciplines covered, but also most engineers practicing biomimetics are specialised in one area. Indeed, those active in biomimetic research are often not specialists in biomimetics specifically, but utilise aspects of biomimetics as a tool for their actual areas of concern. However, it would be desirable that a biomimetic methodology, as proposed, could also be effectively applied in isolated pieces of research.

\subsection{Systematic biomimetics background}

Various efforts have been made towards systematic biomimetics. Most so far have been focused on the systematic transfer of ideas. For instance, databases have been developed for the matching of biological systems to potential engineering applications (Biomimetics Registry Project 2005; Bionics2Space Database 2005; http://people.bath. ac.uk/ensab/TRIZ/). In bionics2space.org (Bionics2Space Database 2005) and the University of Bath's biological effects database (http://people.bath.ac.uk/ensab/TRIZ/), through careful categorisation of the functions of biological systems, biological systems with attractive characteristics can be classified by function to allow a systematic search for potential solutions. While the utility of such strategies is highly dependent on the appropriate functional classification of systems, these represent a first step towards automated tools for biomimetics, in initial problem/solution matching, and a framework for closer collaboration between biologists and engineers.

Originating from Russia, the TRIZ methodology has been proposed as a framework for systematic biomimetics and is related to some of the developments described in the previous paragraph, where careful functional classification is a primary feature. In application to the generation of novel solutions from patents, it has been shown to provide not only reliable descriptions of problems, but can also be used to provide strong indications towards potential solutions. However, for high functionality, this particular option for biomimetic design requires expert interaction to find solutions, and may, therefore, not be ideal for automation (Vincent and Mann 2002; Vincent et al. 2006). Work can also be found concerning general systematic engineering methodology in conjunction with the TRIZ system (de Carvalho and Back 1999). The focus of these previous efforts into systematic biomimetics has been mainly on initial problem/solution matching, rather than systematic distillation of appropriate principles from biological systems and detailed translation and implementation of these principles into an engineered system. 
Further to the above discussion we propose the following requirements for a potential biomimetic methodology with the overriding objective of being a useful tool for the biomimetic engineer: (1) generally applicable across all technical disciplines relevant to biomimetic system design (e.g. those described in Section 3.2); (2) generally applicable across all relevant biological disciplines (i.e. those required for definition of biological system features as described in Section 2); (3) does not, through its application, exclude any possible satisfying solutions and (4) describes the biomimetic process, from input to final design.

Beginning with only the most fundamental of biomimetic concepts, we attempt to build a structure fulfilling these criteria from which the broadest possible range of further options for development may be derived. For example, it is hoped that by building from fundamental components, methodology may be kept general wherever appropriate to allow automation of parts of the process. For this reason, the definitions made in the terminology section are broad. With such a structure, and subsequent elaborations, the following benefits might potentially be provided: (a) a guide to biomimetic engineering for those new to the field; (b) an opportunity to perform a systematic search for biomimetic solutions, accounting for many possible matches, and levels of system abstraction; (c) a tool for the biomimetic engineer, facilitating the tasks required in the design process; (d) a framework for the analysis and improvement of the biomimetic design process and (e) basis for the definition of further tools, potentially for both the improvement and automation of parts of this process.

Challenges in such a definition, summarised from above, include (1) the range of engineering disciplines over which biomimetics can be applied; (2) the range of levels of abstraction possible in translation of the design, where many options are often not obvious and (3) the tendency to perform biomimetics in an ad hoc manner.

\subsection{Process flow: baseline definition}

We now propose a qualitative high-level process flow that can be seen in Figure 2. The process has been designed to include separation of tasks that might be sensibly decoupled and address the desires for generality and systematic search described in the previous sections. While this process is described at a higher level than other approaches to systematic biomimetics discussed in Section 4.2, it attempts to address the entire design process of a system, including detailed design and implementation. The constituent tasks are described below and a hypothetical illustrative example is presented to clarify its possible implementation.

\subsubsection{Input and output}

Input to the process (see Figure 2(a)) may be a biological solution that is observed to perform its function well or in a

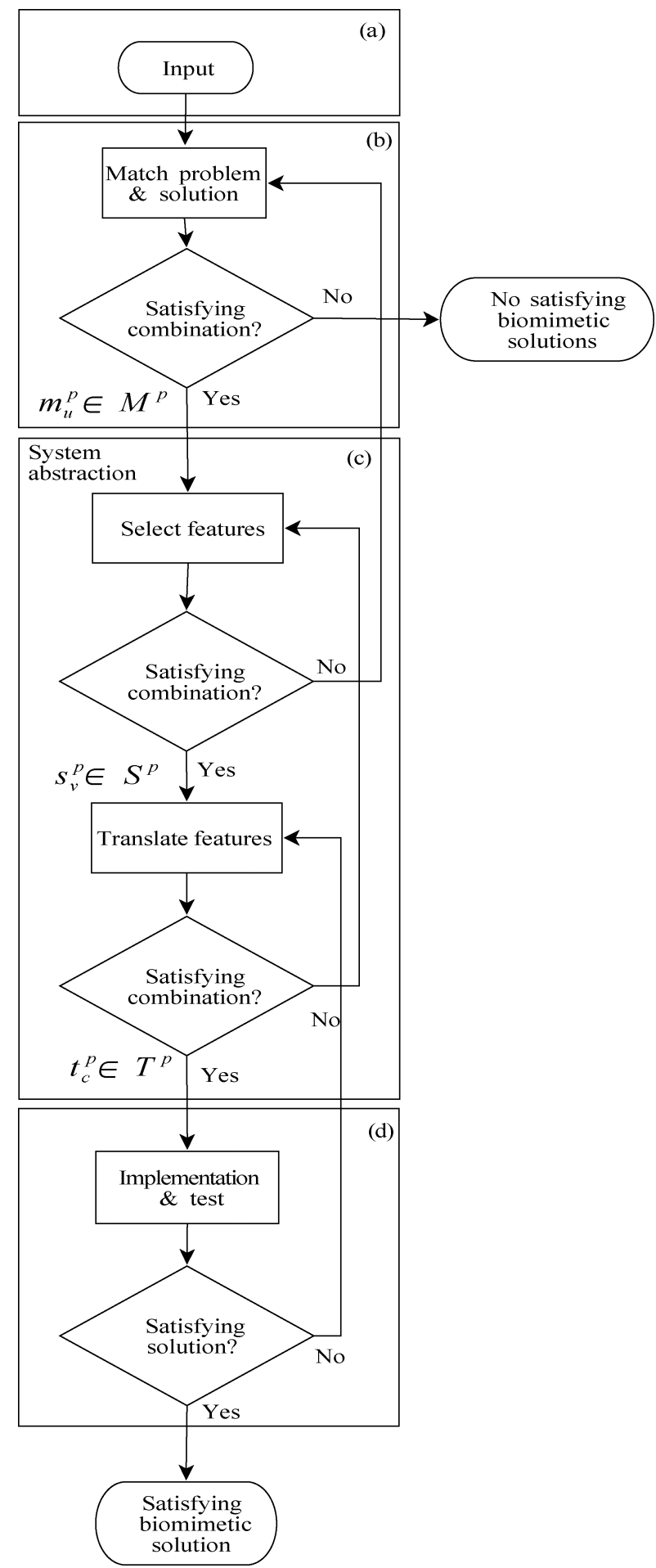

Figure 2. High-level flow-chart for a general, systematic process for biomimetic design.

novel manner from an engineering perspective. It may also be a requirement for a solution to an engineering problem. The output should be a satisfying solution to an engineering problem. For our illustrative example we take as input the 
requirement for a flying device, imagining that no such device exists yet.

\subsubsection{Match problem and solution}

From the input of an appealing biological input, the match problem and solution stage (see Figure 2(b)) would seek to identify an engineering problem that might benefit from application of some subset of principles used in the biological system. The output of this process is a set of potential matches of problem to solution. The approaches to systematic biomimetics described in Section 4.2 mainly address this portion of biomimetic design. For the input of an engineering problem, a biological solution should be sought with potential for application to the whole problem or some required or potential subsystem.

Consider a problem with $i$ potential matches of problem to biological analogue, $m_{i}$, such that the solutions constitute the set $M=m_{1}, \ldots, m_{i}$. This can then be reduced to the set of potentially satisfying solutions $M^{p}=\left\{m_{1}^{p}, \ldots, m_{j}^{p}\right\} \subset$ $M$ through expert analysis or other means. A particular element $m_{u}^{p} \in M^{p}$ is the output of the match problem and slution stage where $1 \leqslant u \leqslant j$. The decision function 'satisfying combinations?' passes the elements $m_{u}^{p}$ while elements are available (see Figure 2). If no values of $m_{u}^{p}$ remain, the 'no' path is chosen and it is concluded that no satisfying biomimetic solutions are available from the given input.

For our illustrative example, we choose to simply consider flying biological systems. Any number of bird, insect and mammalian species might be chosen as a biological analogue, displaying different strategies and capabilities in flight, but for simplicity we choose to consider a generic bird, displaying both flapping and gliding flight, in this instance. The particular system dealing with flight is the wings in this case, which perform several distinguishable functions in providing propulsion, aerodynamic lift and steering, potentially allowing integration of aerodynamic lift with other functions.

\subsubsection{System abstraction - feature selection and translation}

System Abstraction (see Figure 2(c)) and its constituent parts of select features and translate features refer to the process of translating a design into engineering terms, with the result being a concept design for the biomimetic system. This stage may be seen as selection of some set of features to be mimicked followed by the integration of these features into an engineering design using technologies and techniques available to the engineer. System abstraction can therefore be seen as choosing subsets of features of the biological system and then choosing from the many options for translation of each subset. A low level of abstraction might be seen as a design incorporating a high percent- age of identified biological features for translation, with the subsequent translations leading to implementation similar to that of the biological system.

From the input $m_{u}^{p}$, we consider the biological system in the matched combination and define its functions, principles, characteristics and methods (see Terminology section). For a biological system with $k$ functions, $l$ principles, $n$ characteristics and $q$ methods defined respectively, we can define these as the following sets: $F=\left\{f_{1}, \ldots, f_{x}, \ldots, f_{k}\right\}$, where $f_{x}$ is the $x$ th function of a biological system; $P=\left\{p_{1}, \ldots, p_{x}, \ldots, p_{l}\right\}$, where $p_{x}$ is the $x$ th principle of a biological system; $C=$ $\left\{c_{1}, \ldots, c_{x}, \ldots, c_{n}\right\}$, where $c_{x}$ is the $x$ th characteristic of a biological system; $E=\left\{e_{1}, \ldots, e_{x}, \ldots, e_{q}\right\}$, where $e_{x}$ is the $x$ th method of a biological system. We define the set $\mathrm{S}$ as $S=\left\{s_{1}, \ldots, s_{x}, \ldots, s_{a}\right\}$, where $s_{x}$ is the $x$ th combination of subsets of F, P, C and $\mathrm{E}$ and $a=2^{(k+l+c+q)}$, the maximum extent of $S$ considering all combinations of all subsets. Interdependencies between elements of F, P, C and $\mathrm{E}$ as well as expert input should prune the solution space considerably from this number to a set $S^{P}$ with $r$ elements such that $S^{p}=\left\{s_{1}^{p}, \ldots, s_{x}^{p}, \ldots, s_{r}^{p}\right\} \subset S$, where $s_{x}^{p}$ is the $x$ th potentially satisfying combination of subsets of $\mathrm{F}, \mathrm{P}$, $\mathrm{C}$ and E. The output of the stage select features (see Figure 2(c)) is therefore a particular element $s_{v}^{p} \in S^{p}$, where $1 \leqslant v \leqslant r$, a potentially satisfying combination of functions, principles, characteristics and methods. The decision function 'satisfying combinations?' passes the element $s_{v}^{p}$ to the next stage, if available. If no values of $s_{v}^{p}$ remain, the 'no' path is chosen and the procedure returns to the stage match problem and solution to choose another element $m_{u}^{p}$, if available. A similar approach may be taken for the stage translate features (see Figure 2(c)), though sets to be combined to form the output set of potentially satisfying translated system designs will be approaches and technologies available to the particular engineering field involved. We define the set $T^{P}$ as $T^{p}=\left\{t_{1}^{p}, \ldots, t_{x}^{p}, \ldots, t_{b}^{p}\right\}$, where $t_{x}^{p}$ is a potentially satisfying combination of subsets of design approaches and technologies. The output from the translate features stage is $t_{c}^{p} \in T^{p}$, where $1 \leqslant c \leqslant b$ (see Figure 2).

Concerning our illustrative simplified example, let us assume that flapping motion provides propulsion, aerodynamic lift is enabled by the shape of the wings and steering is through both manipulation and orientation of the wings as a whole. In relation to these principles, a characteristic could be that the system functions are provided at relatively low weight though maintaining a strong structure, while another might be that self-healing materials are a method used for robustness of the system. Combination of features in a concept design might consider each individually, while bearing in mind the synergies between them, for instance considering the use of deformations of the wing for both flapping and steering. Implementation of the described process is illustrated in Figure 1 in relation to this hypothetical 
flying device. It can be seen that solutions will be highly dependent to the initial definition of functions, principles, characteristics and methods such that great care should be taken in such definitions.

\subsubsection{Implementation and test}

In the implementation and test stage (see Figure 2(d)), the particular design output from the previous stage, $t_{c}^{p} \in T^{p}$, is implemented. Decoupled design and implementation intend that particular issues of biomimetics need not to be considered in this phase. However, it should be noted that emerging technologies often enable biomimetic design so that consideration of use of new technologies and techniques should be a key element of a systematic search for solutions. Failure to implement a satisfying solution from the design causes the process to return to the translate features stage to select another element $t_{c}^{p}$.

\subsection{Discussion and elaboration}

Taken literally, the process described in Figure 2 will run until a single satisfying solution is found. Other search scenarios incorporating greater solution optimisation might be simply realised from this basis, for instance including assessment of the most promising solutions. As described in Section 2, decoupling of design parameters is seen as being important to a methodical process. As such, any search structure allowing a methodical search for solutions would be valid given effective decoupling of tasks.

Decoupling of the processes involved in this definition has been performed with a view to potential automation. However, these stages can rarely be made completely independent, with careful consideration of interdependencies of system features being important to successful designs. The success of decoupling and the design process in general will be dependent on careful definition of all basic system features, which will rely on expert input. With such careful definitions automation might be feasibly achieved in the generation of elements of the principle solution sets $\mathrm{M}, \mathrm{S}$ and $\mathrm{T}$, ensuring that all possible solutions may be considered. As described in Section 4.3.3, for instance, additional initial definition of relationships between system features might be used to automatically prune solution sets of unfeasible elements. Evaluation of the value of possible solutions, however, seems again to require expert input, though approaches might be considered in weighting of potential solutions, perhaps again through initial definition of feature relationships. For example, where two technologies can be defined as being likely to be complementary in the design of a solution, solution elements containing both might be given higher value in the first instance. Though clearly challenging, with robust and comprehensive description of technologies, it might be envisaged that all potential implementations of a design concept might be suggested through automation, as for example the many methods of synthesizing micro-hair structures illustrated in Sections 5.3.2 and 5.3.3.

The process does not explicitly define a method of interaction for the systematic transfer of research from biology to engineering for the purposes of biomimetics. Four main options may be defined in this context: (1) fostering interaction between biologists and engineers; (2) engineers directing the work of biologists towards areas of interest; (3) biologists directing the work of engineers towards possible systems that might provide attractive solutions and (4) search of biological literature by engineers.

The biomimetic matching databases discussed previously (see Section 4.2) address above-stated issues (1) and (3). While much biological research is already useful in the design of biomimetic systems, two-way communication between the two fields enables the direction of research to areas of particular interest for biomimetic design, which could lead to enhanced solutions. On the other hand, successful biomimetic systems have been realised purely through the review of biological literature.

To give an example of possible progress in the area of automation of system abstraction, one may consider again strategies devised for biomimetic-matching databases, where through appropriate functional classification, an effective automated tool may be devised to give indications of possible solutions. An analogous need may be found in the process of abstracting the biological system where relevant enabling technologies and analytical engineering methods must be found and combined. Through appropriate classification of engineering methods, tools may be envisaged to give initial indications as to the range of possibilities available in the abstraction of biological systems to engineering analogues. The system abstraction stage of Figure 2 is designed with this in mind, being a process usually entirely performed using expert input, which is able to quickly abstract biological concepts to engineering designs. Splitting of this stage into sequential processes, such as feature selection and feature translation, could offer a potential route to a systematic search.

Development of this methodology might make use of previous work towards systematic biomimetics and analogous approaches. For instance, work relating to TRIZ has addressed formal description of systems and solutions that might inform some progress in matching and system abstraction stages of this methodology (Figure 2(b) and (c)). In potential development of automated tools based on this approach, it has been suggested that reasoning approaches used in the artificial intelligence community might have relevance. 


\section{Case study: a climbing robot for use in space}

\subsection{Case study description}

As an example of the use of the general methodology described in Section 4, this section describes biomimetic design of several subsystems with the objective of the design of a climbing robot suitable for operation in space environments. Referring to Figure 2(a), this can be considered as the input to the biomimetic design process, though one could equally consider a particular subsystem as an input problem. The examples described in the following sections demonstrate the design of engineered systems at varying levels of abstraction in their extraction of principles from their biological analogues. Work on these example studies is ongoing (Sameoto et al. 2008; Menon et al. 2008) and as such, while examples are given for differing iterations of the main process, many more iterations for the main objective of a climbing robot for space as well as within each subsystem are possible and would be desirable for the design of a satisfying solution. Reference is made to the design stages illustrated in Figure 2 throughout this section. These examples may be seen as different iterations of the methodology and should illustrate how a broad range of potentially satisfying solutions might be generated through the use of this methodology.

\subsection{Problem description}

Current approaches to adhesion for climbing robots mainly fall into five categories: (1) Negative pressure (Cepolina et al. 2003; Illingworth and Reinfeld 2001; Tummala et al. 1999); (2) Mechanical grip (Bretl et al. 2004; Haynes and Rizzi 2006; Joudrier et al. 2005; Kim et al. 2005; Visentin 2005); (3) Magnetic (Grieco et al. 1998); (4) Electrostatic and (5) Dry adhesive.

Many examples may be found of robots for terrestrial applications using the first three approaches and have been discussed in terms of attractiveness for use in space in previous work (Menon et al. 2007). Broadly speaking, their weaknesses include requirements relating to power for generation and maintenance of adhesion, ambient pressure and climbing surface properties.

Recent research has demonstrated the use of electrostatic force (category-4) for adhesion (Prahlad et al. 2008), similar to dry adhesion in geckos. This approach has been found to be effective for climbing robots on various surfaces, including dusty surfaces. Furthermore, adhesion may be modulated and the technique could be implemented in diverse robotic designs. While not fully assessed for suitability in space, current designs require power to maintain adhesion. The fifth category, dry adhesion, encompasses multiple independent research efforts into this biomimetic technology, one instance of which will form part of the case study presented in Section 5.3.
Therefore, while currently available adhesion strategies are useful in certain circumstances, weaknesses, which make them unfeasible or less desirable for many applications in space, can be identified. As input to the described methodology, we then give as a case study the engineering problem of a climbing robot for space applications.

Many examples of climbing systems may be found in nature, and in particular the phenomenon of dry adhesion utilised in similar ways by several species, such as the gecko and spider that use dry adhesion in conjunction with appropriate kinematic strategies, is of particular interest to implement robust and highly functional climbing behaviour. Discussions of gecko and spider adhesion and climbing strategies may be found in literature (Kesel et al. 2004; Menon et al. 2007; Gasparetto et al. 2008).

\subsection{Adhesion subsystem}

\subsubsection{Match problem and solution}

Referring to Figure 2(b), the match problem and solution stage should be considered from the input of designing a climbing robot for space applications. An initial solution set of biological analogues might include numerous climbing animals including the gecko and the spider.

As discussed in Section 5.2, current strategies have weaknesses or are unsuitable for use in many space applications. It has been shown that the gecko is capable of exerting high adhesion forces mainly generated through van der Waals forces (Autumn 2006). Gecko's feet exhibit the highest density of surface hairs found so far among animal species (Arzt et al. 2003). Dry adhesion is based on contact splitting (Peressadko and Gorb 2004), i.e. the division of the contact area into an enormously increased number of single-contact points between the micro-hairs and the substrate, which generates adhesion (Arzt et al. 2003). In addition, the gecko exhibits rapid robust locomotion on a variety of surfaces including those with high dust loading and is predicted even to maintain adhesion in vacuum. For this iteration the gecko is chosen as a biological analogue to the specified problem given that the features present in the biological system as described would be highly attractive for use in space robotics.

\subsubsection{System abstraction-feature selection and translation}

In this case of the select features stage (see Figure 2(c)) we choose to consider a subset of features involved in the dry adhesion system of the gecko. Numerous efforts have been made in the manufacture of materials with high density of micro-scale hairs, extracting from biology the principle of contact splitting through the use of micro-hairs as described above to produce adhesive force. Approaches have focused on the use of materials such as various polymers 
(Sitti and Fearing 2003), polymer organorods (Northen and Turner 2005) and multi-walled carbon nanotubes (Zhao et al. 2006) in conjunction with fabrication techniques such as electron-beam lithography (Geim et al. 2003), micro/nano-moulding (Glassmaker et al. 2004; Majidi et al. 2004; Sitti and Fearing 2003) and self-assembly (Aksak et al. 2007). In general, the geometrical properties of the biological system are mimicked, while the biological materials used are not extrapolated to synthetic designs because it has been found that adhesion properties are primarily dependent on geometry (Autumn et al. 2002) and materials are chosen for their ability to produce required geometries. Other studies have identified hair features, such as hardness, aspect ratio, angle (Aksak et al. 2007) and tip morphology, (Kim and Sitti 2006) to be important in adhesion and have attempted to manufacture synthetic analogues.

Considered in the frame of the proposed methodology, all these pieces of research could be seen as multiple iterations at differing level of abstraction of the biomimetic design of gecko-inspired dry adhesive for a particular engineering problem, in this case adhesion for a climbing space robot. Iterations at progressively lower levels of abstraction can be seen where more principles from geometric features of gecko hairs are extracted and different routes for translation of these principles may be observed in, for instance, the choice of different materials or manufacturing processes.

Multi-scale compliance is critical in gecko adhesion in maximising surface contact area. Geckos incorporate compliance from the nanoscale level in the smallest hairs on their footpads to millimetre and centimetre scales through conformity in their feet and bodies (Kim et al. 2007). Multiscale conformity is provided in the hair structures themselves that incorporate branching structures with elements of differing scale. In addition, therefore, to the production of uniform small-scale fibres, it is desirable to manufacture such multi-scale fibre structures.

A low-level abstraction was considered in this stage (see Figure 2(c)) by selecting more features of the biological system and designing compliance at multiple scales (Sameoto et al. 2008). Nanoscale features are the most difficult to replicate using traditional microfabrication technologies. In some of the aforementioned research into the fabrication of dry adhesives, manufacture has been addressed with moulding (Geim et al. 2003; Gorb et al. 2007; Kim and Sitti 2006) or dry etching (Geim et al. 2003), but as the minimum feature sizes shrink, standard lithography is no longer capable of defining structures. Early attempts at nanocasting polymers were unsuccessful as separating the fibres from their moulds required dissolving the mould, which resulted in clumped and ineffective fibres (Campolo et al. 2003; Sitti and Fearing 2003). More recently, the nanostructuring of polymers has been demonstrated using dry fabrication techniques, either integrated with silicon microstructures (Northern and Turner 2005) or demonstrated independently of any microscale features (Chen et al. 2007; Nabesawa et al. 2007). In earlier work, a $\mathrm{O}_{2}$ or $\mathrm{O}_{2} / \mathrm{CF}_{4}$ reactive ion etch (RIE) was used which the authors claimed produced nanorods due to an electric field gradient across a photoresist layer left behind on glass features. Other researchers have reported similar structures in poly(methyl methacrylate) PMMA (Nabesawa et al. 2007) or polydimethylsiloxane (PDMS) (Chen et al. 2007) layers using an $\mathrm{O}_{2} / \mathrm{CF}_{4} \mathrm{RIE}$, but have attributed their formation to micromask generation during the etching process. Micro-masking is a well-known problem in silicon reactive ion etching and occurs when masking materials are sputtered and redeposited, which results in uneven etching and rough surfaces. This micromasking can occur for a number of contaminants, but gold has been identified as the material most likely to produce this effect (Jansen et al. 1996).

Previous demonstrations of micromasking for the production of nanoscale polymer features have used either glass (Chen et al. 2007) or aluminium (Nabesawa et al. 2007) as the micromask-generating material. For this attempt, mask material was planned to be located above the material to be etched. Relatively thick polymer columns defined on top of a micromask-generating material were proposed to mimic larger hair structures. Using micromasking to create nanoscale structures on these columns was then proposed to mimic branching-compliant hairs at this smaller scale.

\subsubsection{Implementation and test}

5.3.3.1. Micro-structures. Microscale posts are formed by casting a two-part elastomer in a mould fabricated using high-aspect ratio lithography of a thick photoresist layer. Using low-modulus elastomers, like silicones or polyurethanes, greatly reduces the complexity of the structures required to achieve a sticky adhesive. Due to the large size of the adhesive areas required to be effective for a climbing robot, efforts are focussed on achieving maximum yield and long-term reliability. Future work for space applications will use harder materials with smaller features, once the necessary processing knowledge is worked out. In the meantime, early designs are produced primarily using a common silicone available from Dow Corning (Sylgard 184), which has relatively well-known material properties and is easy to work with. Its very low surface energy offers the potential of easy cleaning, but at the expense of maximum expected adhesion from van der Waals forces. Existing designs for microposts include the commonly used circular cross-section designs (Figure 3(a)), for general purpose adhesion, as well as novel micro-treads (Figure 3(b)), which are designed to provide good frictional adhesion in specific orientations, but minimal adhesion when loaded in peel. The micro-tread is designed to accelerate the rate at which collapsed posts become unstuck, which should eliminate some of the adhesion loss with time. Adding a mushroom-shaped tip to these adhesive microposts will 


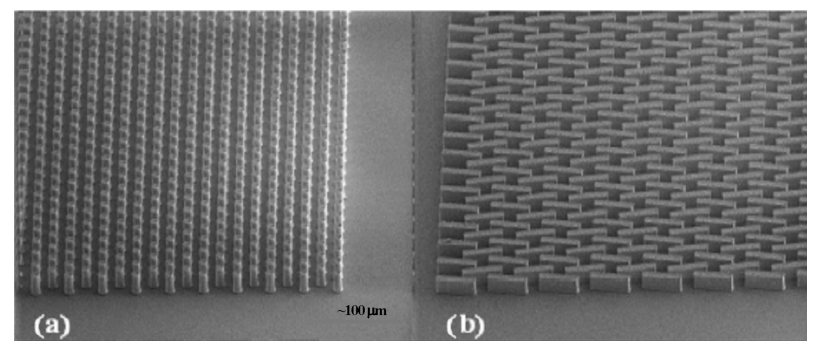

Figure 3. Microstructures fabricated in Sylgard 184 designed for use with climbing robots. (a) circular cross-section posts designed for general adhesion, (b) a novel micro-tread design to be used for track robots.

increase the contact area and should dramatically improve the adhesive properties of these designs.

5.3.3.2. Nano-structures. For harder materials, the aspect ratio of posts must be much higher and the minimum feature size much smaller to achieve similar adhesive forces. This greatly complicates fabrication, as casting techniques are no longer appropriate. A relatively stiff polymer can have its surface altered in a way that mimics the finest features of a gecko's toe hairs through a naturally occurring process during reactive ion etching. The polymer chosen was the epoxy-based, negative-tone photoresist SU8 (Lorenz et al. 1997). This commonly used micro-electromechanical system (MEMS) polymer is directly photopatternable using near ultraviolet light, and is mechanically, chemically and thermally stable after crosslinking (Lorenz et al. 1997).

The etching of SU-8 was completed with a combination of $\mathrm{O}_{2}$ and $\mathrm{CF}_{4}$ gases at different times and pressures. Lower pressures have been identified as beneficial for achieving high-aspect ratio grass structures (Nabesawa et al. 2007), while the effect of gas ratio on the development of SU8 grass morphology has not been reported previously. The minimum etching pressure possible with the RIE equipment used was 60 mTorr.

Oxygen will etch nearly any polymer, while $\mathrm{CF}_{4}$ has been identified as a gas that can partially passivate polymer sidewalls (Nabesawa et al. 2007) and help enhance grass formation. Earlier reports of nanohair formation also used relatively long etching times (Chen et al. 2007; Nabesawa et al. 2007), but the effectiveness of gold as a micromasking material means that grass evolution can occur much faster.

After etching, samples were observed using a scanning electron microscope. The hairs were characterised by average size, area percentage and height for each of the etching conditions. National instruments vision builder for automated inspection was used to collect these values from the SEM images. Examination of SU-8 etched without the presence of gold revealed that hair formation only occurred if a micromasking material was present (Figure 4).

\subsection{Climbing, steering and transiting through different gradients}

To provide contrasting examples, this section and Section 5.5 present brief examples of the biomimetic design of climbing systems that may take advantage of synthetic dry adhesives in our overall objective of designing a climbing robot suitable for operate space environments (see Section 5.1).

Let us hypothesise that all subsequent options from the element $s_{v}^{p} \in S^{p}$ chosen in Section 5.3.2 have been exhausted, remembering that only those features relating directly to dry adhesion of gecko were selected. Let us also hypothesise that a satisfying synthetic dry adhesion was eventually manufactured. In the last stage of the procedure presented in Figure 2(d), the stage 'satisfying solution?' yields a negative result as the overall objective of designing a climbing robot is not achieved, although new bio-inspired adhesives were developed. The backward loop leads again

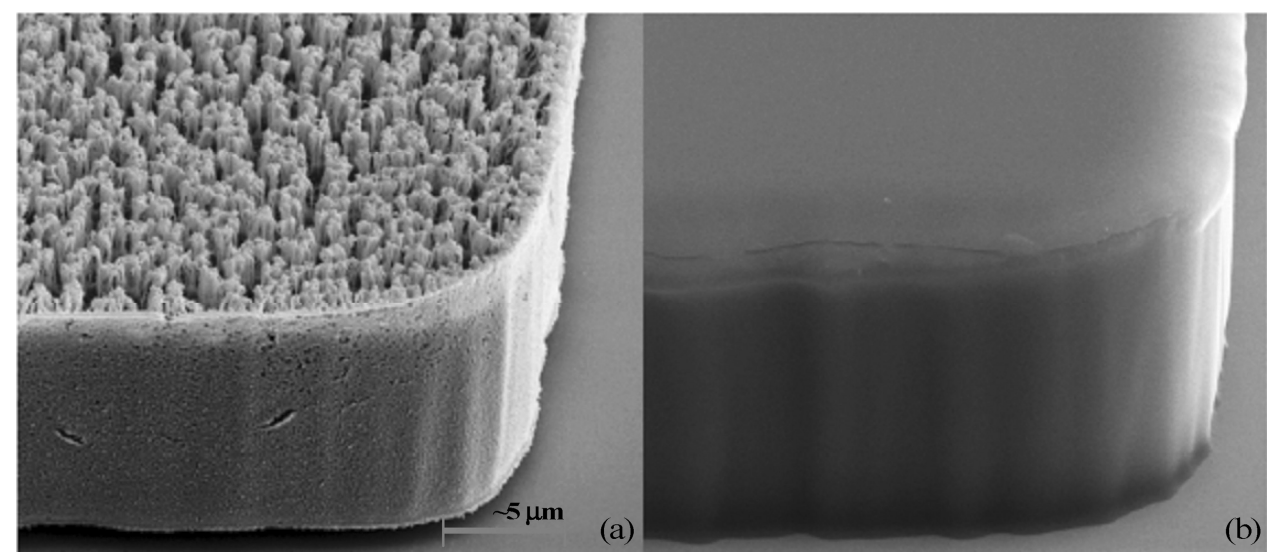

Figure 4. SEM images of SU-8 posts (a) etched on a gold-coated substrate, (b) etched with all sources of micromasking removed. SU-8 adhesion to gold is relatively poor, and some posts separate from the substrate (a). 


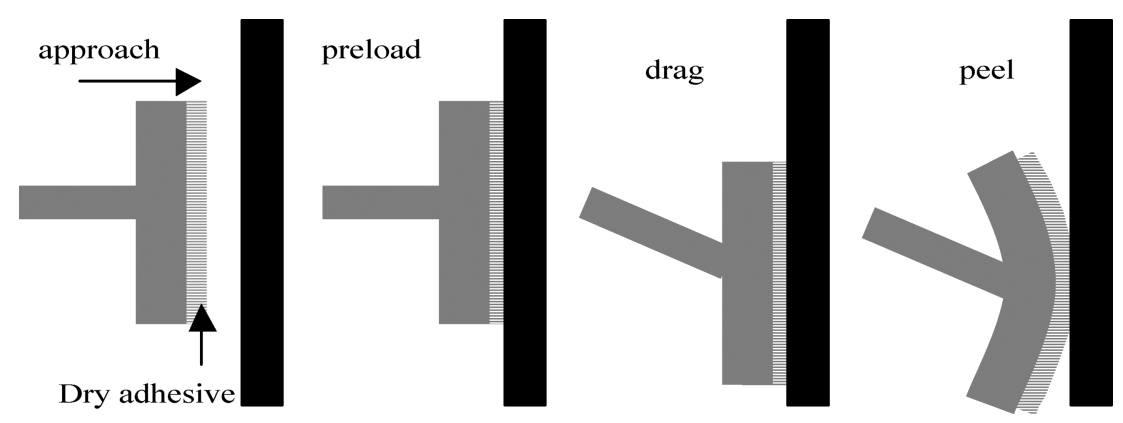

Figure 5. Idealised adhesion and detachment strategy of a gecko foot.

to the stage select features. In this new iteration, another choice of $s_{v}^{p} \in S^{p}$ is performed while the element $m_{u}^{p}$ remains the same - the gecko's features will again be considered as source of inspiration to fulfil the main engineering objective.

\subsubsection{System abstraction-feature selection and translation}

An idealised view of gecko foot motion is shown in Figure 5. Adhesion between footpad hairs and the surface is achieved as the footpads are preloaded against the surface, allowing the hairs to conform to the surface and maximise contact area. Subsequently, a twisting motion of the foot from the tip is used in the peeling phase to free the adhesive from the surface, where the pad gets separated from the surface at a critical angle of about $30^{\circ}$, requiring minimal force. Features relating to this behaviour are selected for this iteration (see Figure 2(c)). Taking the principles from nature of adhesion through the preloading of a pressure sensitive adhesive (PSA) and detachment with minimal force through a peeling motion, a high level of abstraction was used to develop an engineering system. A track vehicle was implemented as its design intrinsically allows preloading and peeling though revolution of its tracks.

\subsubsection{Implementation and test}

With regards to the implementation and test stage (see Figure 2(d)), the system presented in Figure 6 was developed from the preceding design. The prototype has a modular structure, each module consists of six wheels, two DC motors with gearboxes, four potentiometers and two belts (tracks) to which commercial adhesives can be fixed. The chassis was built by using rapid-prototyping manufacturing to take advantage of solid freedom fabrication. Belts are kept in tension by using a spring-loaded piston, which constantly preloads the front wheels. The plane defined by the centres of the four front wheels is inclined at about $45^{\circ}$ to the flat surface - this allows easy transit between surfaces at different angles. Transition between surfaces is also facilitated by the modular design - modules are connected by a spring-damping system that transmits forces among the modules.

The miniaturised track vehicle is inherently suited to preloading and peeling adhesives as the belts continually keep contact with the surface and are peeled off during their revolution - a proper selection of the radius of the rear wheel allows tuning of the peeling force. The modular design of the system also allows overcoming simple obstacles without losing traction. However, this system presents some drawbacks, which prevent its use in unstructured scenarios. Steering is achieved by controlling the two belts of a module with two different velocities; sliding between belt and surface therefore occurs while turning. Although steering during climbing is still feasible, sharp corners should be avoided to prevent falling. Another intrinsic issue of this track vehicle concerns vibration damping on uneven terrains; the front wheels hit obstacles with uncontrolled trajectories and belts continuously impact surface asperities.

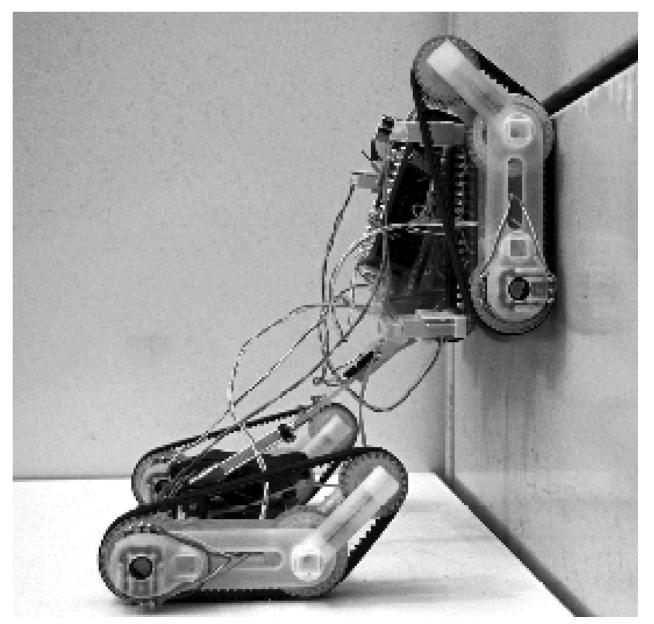

Figure 6. Modular miniaturised track vehicle (this system was developed in Menrva group at Simon Fraser University by C. Gittens, D. Goundar, D. Law and J. Minor, under the supervision of Prof. C. Menon). 
Therefore, although the system outperforms most of the climbing mechanisms on flat surfaces, it is not ideal for uneven terrains as vibrations, which are one of the main causes of adhesion detachment in climbing robots, are generated and not well-suppressed.

\subsection{Highly mobile gait}

\subsubsection{Match problem and solution}

Let us hypothesise that during the iteration, options for the gecko match $m_{u}^{p}$ (see Figure 2(c)) are exhausted so that the process described in Figure 2 returns to the match problem to solution stage to choose another matching element $m_{u}^{p}$, in this case the spider. Also using dry adhesives, the spider is able to exhibit highly agile movement with eight legs each with up to $10^{\circ}$ of freedom. Relatively, even angular distribution of the legs around a central prosoma helps enable robust and agile surface gradient transition to be relatively direction independent (Quian, Zhang, and Ma 2007).

\subsubsection{System abstraction-feature selection and translation}

For this select features stage (see Figure 2(c)), we, for this iteration, choose to take into account the spider features of multiple legs with roughly even angular distribution around a central body as well as multi-joint legs with multiple degrees of freedom (DOF). In the translation of these principles to an engineering design, fewer legs, leg joints and DOF are chosen for design practicality and potential for miniaturisation, leading to a comparatively intermediate level of abstraction in relation to the previous examples. In fact, a six-legged robot with six DOFs per leg was designed and implemented, with 18 miniaturised revolute motors for actuation. Symmetric angular distribution of the legs around the centre of the robot enables direction-independent surface gradient transition. Gait design was based on the team's observations of spider foot detachment, where a simple leg extension is used for detachment.

The gecko's attachment and detachment strategy is again considered for the problem of foot design, as in the previous section considering preloading and peeling phases. The engineering problem can be considered to include integration into the spider-based design described above and, in particular, use of the spider's leg-extension detachment strategy. Compliant feet were envisaged, with three passive and compliant DOFs. Again this can be considered to be a translation at intermediate level of abstraction, reducing the both active and passive compliance of gecko feet to simply passive compliance in the robotic system. Attachment of the leg to one end of the foot allows a pulling component of force during the outward arc of leg movement to detach the foot with a peeling behaviour, while appropriate stiffness of

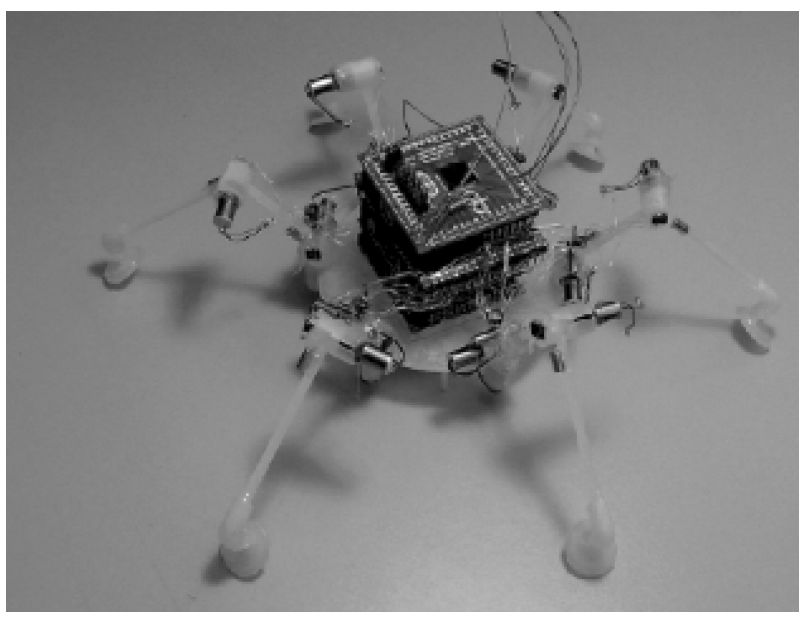

Figure 7. The Abigaille-I prototype.

the foot material allows preloading of the unattached end of the foot during the attachment phase. The compliant foot in conjunction with attached synthetic micro- and nano-fibre dry adhesives as described in Section 5.3 enables passive multi-scale compliance from the foot scale to that of the smallest fibres.

\subsubsection{Implementation and test}

For the compliant feet, a mix of PDMS and a cross-linking agent was used, utilising moulds produced by a rapid prototyping 3-D printer and undergoing degassing under vacuum and baking. It is possible for stickier PDMS or synthetic dry adhesives to be bonded to the bottom of the feet. These were integrated into the robotic implementation of the first iteration named Abigaille-I (Menon et al. 2008), as shown in Figure 7.

Analysis of the robot leg kinematics was performed, particularly important in relation to the identification of suitable foot trajectories in enabling suitable foot attachment and detachment. For this purpose, direct kinematics gave the position of the leg-end effectors as a function of leg joint variables and inverse kinematics gave the joint variables as a function from the position of the end-effectors. Using these results, kinematics of the robot as a whole were calculated considering a 'crab gait'. These kinematic models were verified through multi-body simulations using commercial software.

To investigate optimal leg angle for this system, a simple robot with three symmetrically distributed legs (tripod) was constructed with each leg controlled by one servo-motor, with varying angle $\alpha$ between the leg and surface. PDMS feet were used. With a mass of $97 \mathrm{~g}$, this tripod was placed gently on a smooth horizontal surface. A force normal to the surface was then used to detach the robot for varying values of the angle $\alpha$. Optimal leg angles were found to 
be between $35^{\circ}$ and $45^{\circ}$, requiring a force of $0.195 \mathrm{~N}$ for detachment, almost double the weight of the robot.

\section{Conclusions and future work}

The usefulness of biomimetic design as applied to robotics has been discussed, particularly with respect to space applications. With reference to the compelling attributes displayed by many biological systems in performing useful tasks and the potential transfer of these attributes to robotics through biomimetic design, it has been proposed that this maturing discipline would benefit from accepted methodical practices in its application. A systematic, generalised methodology has been proposed, along with suggestions for its useful development. Examples of ongoing biomimetic research for a robotic system for use in space have been presented with reference to the proposed methodology, illustrating the potential implementation of a systematic search for biomimetic solutions to engineering problems. The biomimetic systems presented in the paper provide a snapshot of ongoing work and will require many more biomimetic design iterations to produce satisfying solutions for our objectives. In the frame of synthetic dry adhesives, current implementations are not (as their biological analogues are) durable, self-healing or self-cleaning, issues that would need to be addressed for feasible use in most robotic applications. The latter two cases represent possible avenues for systems able to take advantage of synthetic dry adhesives, though many such systems could be envisaged or are the subjects of ongoing research. Such prototype biomimetic designs can be considered as individual iterations in systematic searches for biomimetic design solutions.

\section{Acknowledgements}

Thanks are extended to the numerous students in Menrva group that contributed to the development of the prototypes. This work was financially supported by the Natural Sciences and Engineering Research Council of Canada (NSERC) and the European Space Agency (ESA).

\section{References}

Aksak B, Murphy M, Sitti M. 2007. Adhesion of biologically inspired vertical and angled polymer microfiber arrays. Langmuir, 23:3322-3332.

Arzt E, Gorb S, Spolenak R. 2003. From micro to nano contacts in biological attachment devices. PNAS USA, 100(19):1060310606.

Autumn K. 2006. Properties, principles, and parameters of the gecko adhesive system. In Smith A and Callow J, editors. Biological adhesives. Berlin-Heidelberg: Springer. p. 225255.

Autumn K, Sitti M, Liang YA, Peattle AM, Hansen WR, Sponberg S, Kenny TW, Fearing R, Israelachvili JN, Full RJ. 2002.
Evidence for van der waals adhesion in gecko setae. PNAS, 99: 12252-12256.

Karen F, Ayre M, Lan N, editors. 2006. Biomimetic engineering for space applications. ESA Publications Division (ESA SP; 1297. 0379-6566).

Biomimetics Registry Project. 2005. http://www.biomimetic sregistry.net/; [cited 2006 Sep 15].

Bionics2Space Database. 2005. http://www.bionics2space.org; [cited 2006 Sep 15].

Bretl T, Rock S, Latombe JC, Kennedy B, Aghazarian H. 2004. Free-climbing with a multi-use robot. International Symposium on Experimental Robotics (ISER), Singapore.

Campolo D, Jones S, Fearing RS. 2003. Fabrication of gecko foot-hair like nano structures and adhesion to random rough surfaces. Presented at Nanotechnology 2003, San Francisco, CA.

Cepolina F, Michelini RC, Molfino RM, Razzoli RP. 2003. Gecko, a climbing robot for walls cleaning. ASER03, 1st International Workshop on Advances in Service Robotics, 2003 Mar 13-15, Bardolino, Italy.

Chen M-H, Hsu T-H, Chuang Y-J, Chen P-H, Tseng F-G. 2007. Self-formed high-aspect-ratio polymer nanopillars by RIE. Proceedings of the Transducers 2007 conference, Lyon, France, 1:563-566.

de Carvalho MA, Back N. 1999. TRIZ methodology and its use in systematic engineering design. Anais do COBEM '99 (Congresso Brasileiro de Engenharia Mecânica). Águas de Lindóia.

ESA. 2005. Biologically inspired solutions for robotic surface mobility. Prime contractor: University of Helsinki, Finland. Contract No. AO4532/18155/04/NL/MV.

ESA. 2005. Bionics \& space system design. Prime Contractor: Surrey University. Contract No. AO/1-4469/03/NL/Sfe.

ESA. 2006. Biologically inspired joints for innovative articulations concepts. Prime Contractor: University of Bologna, Italy. Contract No. AO18911/05/NL/MV.

Gasparetto A, Vidoni R, Zanotto V, Brusa E. 2008. Attaching mechanisms and strategies inspired by spiders' legs. Ariadna Final Report (06/6201). European Space Agency.

Geim AK, Dubonos SV, Grigorieva IV, Novoselov KS, Zhukov AA, Shapoval SY. 2003. Microfabricated adhesive mimicking gecko foot-hair. Nat Mater. 2: 461-463.

Glassmaker NJ, Jagota A, Hui CY, Kim J. 2004. Design of biomimetics fibrillar interfaces: 1. making contact. J R Soc Interface. 1(1):23-33.

Gorb SN, Sinha M, Peressadko A, Daltorio KA, Quinn RD. 2007. Insects did it first: a micropatterned adhesive tape for robotic applications. Bioinspiration \& Biomimetics. 2:S117-S125.

Grieco J, Armada M, Gonzalez de Santos P, Prieto M. 1998. A sixlegged climbing robot for high payloads. Proceedings of the 1998 IEEE International Conference on Control Applications, Trieste, Italy. 1, 446450.

Haynes G, Rizzi A. 2006. Gait regulation and feedback on a robotic climbing hexapod. Proceedings of Robotics: Science and Systems, 2006, Aug; Philadelphia.

Illingworth L, Reinfeld D. 2001. The vortex attractor. Vortex Holding Company, United States Patent and Trademark Office, USA. US Patents \#6565321 and \#6497553,

Jansen H, Gardeniers H, Boer MD, Elwenspoek M, Fluitman J. 1996. A survey on the reactive ion etching of silicon in microtechnology. J Micromech Microeng. 6: 14-28.

Joudrier L, Didot F, Kapellos K. 2005. Eurobot: use of the muroco formal approach for activity analysis, programming and control. Proc. of The 8th International Symposium on Artifical Intelligence, Robotics and Automation in Space-iSAIRAS; 
2005, Sep 5-8; Munich, Germany. (ESA SP-603, August 2005).

Kesel A, Martin A, Seidl T. 2004. Getting a grip on spider attachment: an AFM approach to microstructure adhesion in arthropods. Smart Mater Struct. 13(3): 512-518. (Epub 20 Apr 2004) doi: 10.1088/0964-1726/13/3/009.

Kim S, Asbeck A, Provancher W, Cutkosky MR. 2005. SpinybotII: climbing hard walls with compliant microspines. IEEE ICAR; 2005, July 18-20; Seattle, WA,.

Kim S, Sitti M. 2006. Biologically inspired polymer microfibers with spatulate tips as repeatable fibrillar adhesives. Appl Phys Lett. 89(26): 26911-26913.

Kim S, Spenko M, Trujillo S, Heyneman B, Mattoli V, Cutkosky MR. 2007. Whole body adhesion: hierarchical, directional and distributed control of adhesive forces for a climbing robot. IEEE ICRA 07; 2007, April.

Lorenz H, Despont M, Fahrni N, LaBianca N, Renaud P. 1997. SU-8: a low-cost negative resist for MEMS. J Micromech Microeng. 7: 121-124.

Majidi C, Groff R, Fearing R. 2004. Clumping and packing of hair arrays manufactured by nanocasting. Proceedings of ASME International Mechanical Engineering Congress and Exposition 2004, 579-584.

Menon C, Broschart M, Lan N. 2007 Biomimetics and robotics for space applications: challenges and emerging technologies. Workshop on Biomimetic Robotics at ICRA, Rome, Italy.

Menon C, Li Y, Sameoto D, Martens C. 2008. Abigaille-I: towards the development of a spider-inspired climbing robot for space use. IEEE RAS/EMBS International Conference on Biomedical Robotics and Biomechatronics, Scottsdale, Arizona, USA.

Menon C, Lira C 2006. Active articulation for future space applications inspired by the hydraulic system of spiders. J Bioinspiration \& Biomimetics, IOP, 52-61.

Menon C, Murphy M, Sitti M, Lan N. 2007a. Space explorationtowards bio-inspired climbing robots. In Habib MK, editor. Bioinspiration and robotics - walking and climbing robots. Vienna, Austria: Advanced Robotic Systems International and I-Tech, 544.

Menon C, Seidl, T, Broschart, M. 2007b. Biomimetic approach to advanced space missions. Proceedings of the 5th IAA Symposium on Realistic Near-Term Advanced Scientific Space Missions, International Academy of Astronautics. pp. 63-68.

Michelson R, Naqvi M. 2003. Extraterrestrial flight. In Proceedings of von Karman Institute for Fluid Dynamics RTO/AVT lecture series on low reynolds number aerodynamics. Brussels, Belgium.

Nabesawa H, Hitobo T, Wakabayashi S, Asaji T, Abe T, Nakatani I, Seki M. 2007. Polymer surface morphology control of microfluidic devices. Transducers 2007 conference, Lyon, France, 1:199-202.

Northen MT, Turner KL. 2005. A batch fabricated biomimetic dry adhesive. Nanotechnology, 16:1159-1166.

Peressadko A, Gorb, SN. 2004. When less is more: experimental evidence for tenacity enhancement by division of contact area. J Adhes. 80: 247-261.

Prahlad H, Pelrine R, Stanford S, Marlow J, Kornbluh R. 2008. Electroadhesive robots - wall-climbing robots enabled by a novel, robust, and electrically controllable adhesion technology. Proceedings of Robotics and Automation, 2008, ICRA 2008. IEEE International Conference, 2008 May 19-23.

Quian J, Zhang Z, Ma L. 2007 . Gait programming for multi-legged robot climbing on walls and ceilings. In Habib MK. editor. Bioinspiration and robotics - walking and climbing robots. Vienna, Austria: Advanced Robotic Systems International and I-Tech. p. 544

Sameoto D, Li Y, Menon C. 2008. Micromask generation for polymer morphology control: nanohair fabrication for synthetic dry adhesives. Proceedings of International Conference on Modern Materials \& Technologies; 2006, June 04-09; Acireale, Italy;

Sitti M, Fearing R. 2003. Synthetic gecko foot-hair micro/nanostructures as dry adhesives. J Adhes Sci Technol. 17(5):1055-1074

Tummala R, Mukherjee R, Aslam D, Xi N, Mahadevan S, Weng J. 1999. Reconfigurable adaptable micro-robot. Proceedings of the IEEE Conference on Systems, Man, and Cybernetics (SMC), 1999 Oct 12-15; Tokyo, Japan.

Vincent JFV, Bogatyreva OA, Bogatyrev NR, Bowyer A, Pahl A-K. 2006. Biomimetics - its practice and theory. J R Soc Interface, 3:471.

Vincent J, Clift S, Menon C. 2007. Biomimetics of campaniform sensilla: measuring strain from the deformation of holes. J Bionic Eng. 4(2): 63-76 (Elsevier).

Vincent JFV, Mann DL. 2002. Systematic technology transfer from biology to engineering. Philosophical transactions of the Royal Society of London Series a-mathematical physical and engineering sciences. 360(1791). pp. 159-173. (1364-503X).

Visentin G. 2005. Robotic programmes and applications at ESA present and perspectives. i-SAIRAS 2005-The 8th International Symposium on Artificial Intelligence, Robotics and Automation in Space. B. Battrick, editor. European Space Agency (ESA SP-603). Published on CDROM, p.1.1; publication date: August 2005

Zhao Y, Tong T, Delzeit L, Kashani A, Meyyappan M, Majumdar A. 2006. Interfacial energy and strength of multi-walled carbon-nanotube-based dry adhesive. J Vac Sci Technol, B: Microelectron Nanometer Structs Process, Meas, Phenom. 24:331-335 

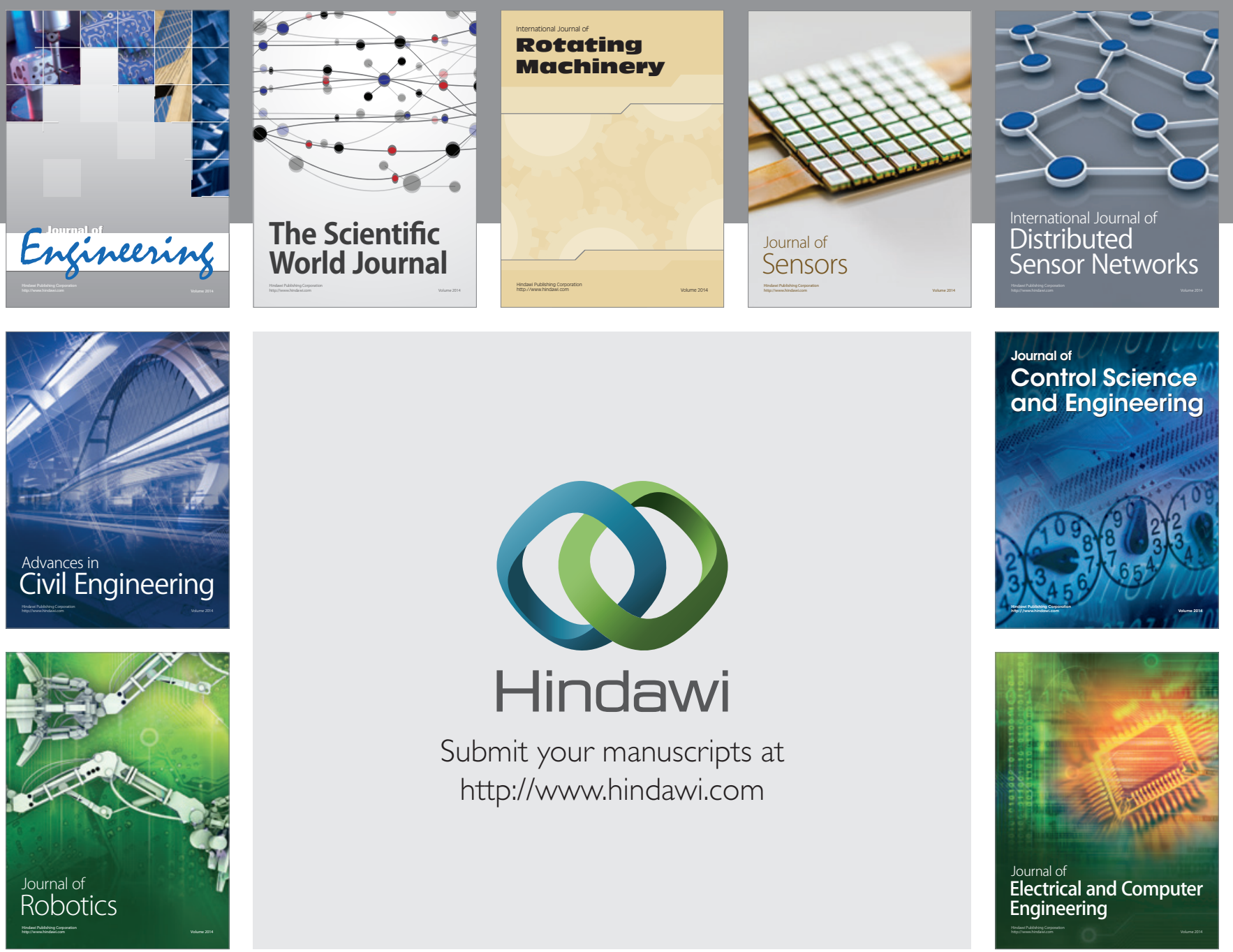

Submit your manuscripts at

http://www.hindawi.com
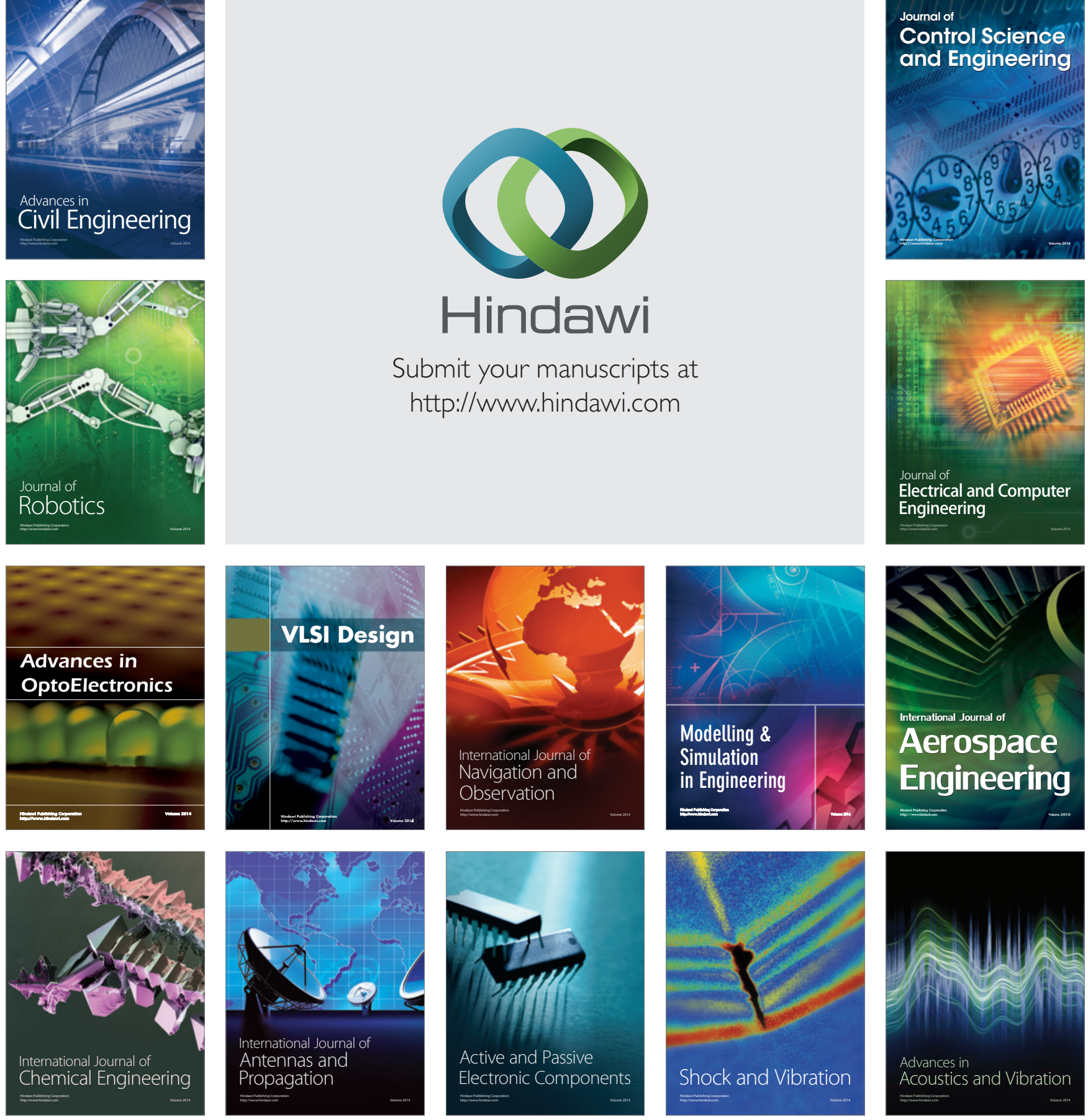